\title{
DESIGN OF EXPERIMENTAL SETUP FOR INVESTIGATION OF CAVITATION BUBBLE COLLAPSE CLOSE TO A SOLID WALL
}

\section{INTRODUCTION}

Cavitation bubble collapse is associated with a number of well-known effects causing erosion of solid surfaces of the liquid flow container [1]. In cavitating liquid flows with travelling bubbles, the number of cavitation impacts on the solid surface is large and their location is random, which makes it impossible to study individual bubbles. Therefore most researchers turn their attention at a single bubble collapse under controlled conditions. The knowledge of the impact force of a single collapsing bubble is important for successful prediction of bubble collapse aggressiveness (also called "erosion potential"). In such experiments, bubbles are usually produced by lasers or spark generators. The deficiency of the approach based on heat-induced liquid breakdown lies in the difficulty to reliably define the initial bubble content. Lasers and spark generators produce high-temperature plasma. The behaviour of such bubbles is supposedly different from the behaviour of bubbles generated by pressure drop under hydrodynamic conditions. The situation is further complicated by the presence of other effects related to violent plasma dynamics, which can strongly influence the experimental results [2].

\footnotetext{
- Miloš Muller, Faculty of Mechanical Engineering, Technical University of Liberec, Hálkova 6, 46117, Liberec 1, Czech Republic, milos.muller@tul.cz

Patrik Zima, Department of Thermodynamics, Institute of Thermomechanics, v. v. i., Academy of Sciences, Dolejškova 5, 18200 Praha, Czech Republic, zimap@it.cas.cz

Jiri Unger, Faculty of Mechanical Engineering, Technical University of Liberec, Hálkova 6, 46117, Liberec 1, Czech Republic, jiri.unger@tul.cz

Martin Živný, Faculty of Mechanical Engineering, Technical University of Liberec, Hálkova 6, 46117, Liberec 1, Czech Republic, martin.zivny@tul.cz
} 
The aim of our effort is to prepare a setup for experimental investigation of the collapse of a single bubble generated and maintained under conditions typical for bubbles travelling inside water pumps. These conditions are characterized by the bubble content consisting of water vapour and air at normal temperature and the bubble initially in equilibrium with the surrounding liquid whose dynamics is controlled by pressure variation.

The main objective of the future experimental studies is to measure bubble impact loads for different initial bubble radii, standoff distances (distance of the bubble from the wall) and pressure variations inside the chamber. Such information can be then used to improve our current models for numerical prediction of cavitation bubble collapse aggressiveness in 3D pump flows [3].

\section{EXPERIMENTAL SETUP}

Figure 1 shows the schematic view of the experimental setup. The core component of the system is the cubic chamber 1 whose body is made of Dural. All side-walls except the one attached to the actuator 8 have Plexiglas windows. The bubble generator (assembly of items 2-6) is located at the bottom of the chamber. The detail of the generator is shown in the inset of Figure 1. The generator consists of the Teflon plate 2, Teflon ring 3, copper cylinder 4 , Teflon holder 5 and electric cartridge heater 6 . Teflon was chosen for

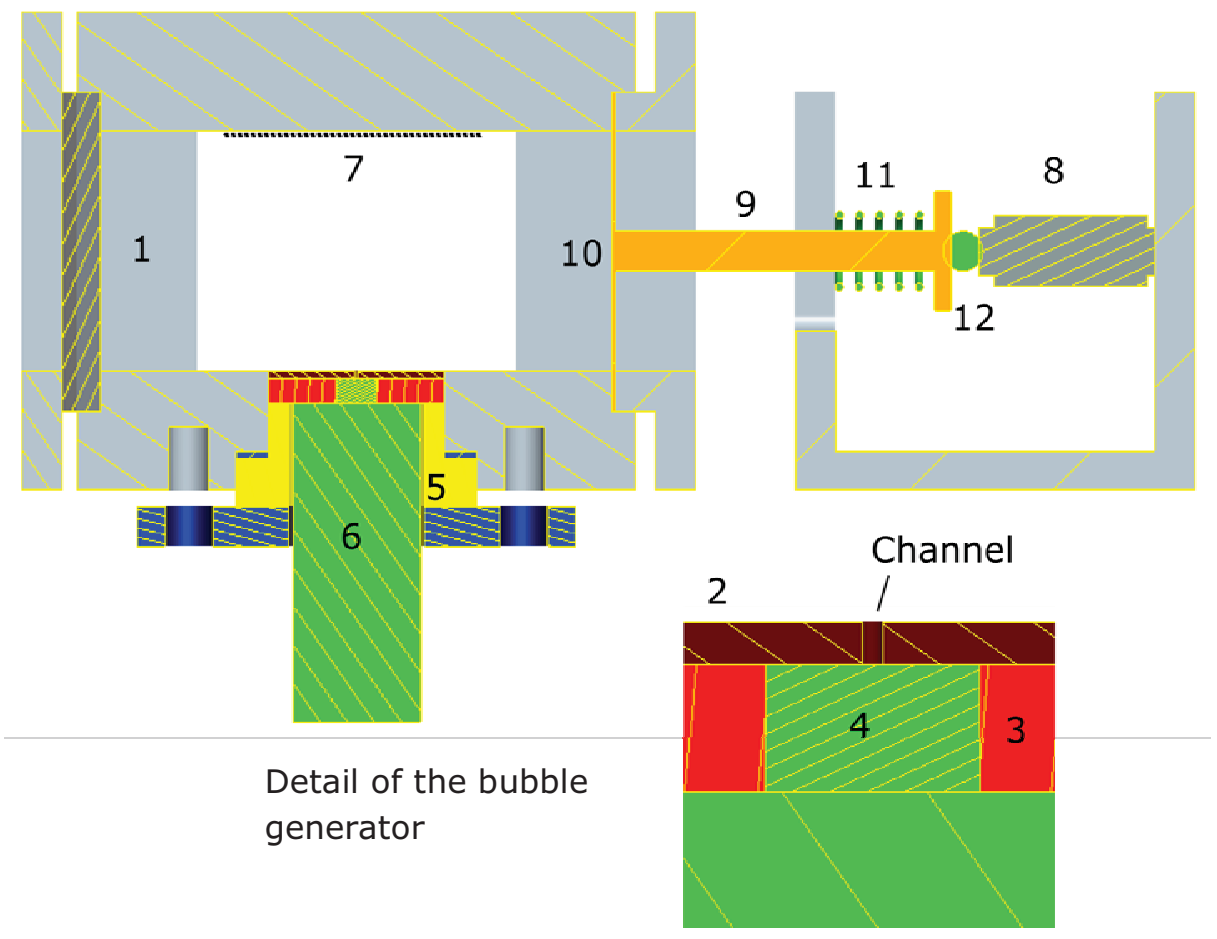

Figure 1. Chamber setup

its low heat conductivity and high temperature resistance. Several replaceable bubble generators with different channel diameters have been manufactured in order to obtain different initial bubble radii. The PVDF piezoelectric transducer (film) 7 for the impact load measurement [4] is glued to the upper wall of the chamber. The piezoelectric 
actuator P-602.308 (Physik Instrumente) 8 for the generation of the pressure pulse is connected through the beam 9 to the copper wall 10, which serves as the excitation element for the pressure pulse generation. Because the actuator can only sustain positive pressure loads, it is connected to the bar via a sphere to reduce the bending load exerted on the actuator. For this reason, the unload is provided by the spring 11 . The resonant frequency of the actuator element is around $450 \mathrm{~Hz}$, which limits the frequency of the generated pulses. The blocking force of the actuator is $220 \mathrm{~N}$ and the maximum displacement is $310 \mu \mathrm{m}$. For a movable wall with dimensions $0.02 \times 0.02 \mathrm{~m}$ the force will correspond to the maximum pressure 5.5 bar. It is important to keep in mind that the resonant frequency of the actuator will vary with the load. The use of the spring also partially reduces the force provided by the actuator.

\section{MeAsurement}

The scheme of the working principle of the system is given in Figure 2. The system is controlled by the LabView application connected to the PXI box. The measurement starts with heating of the cartridge heater. Water inside the channel is evaporated and the bubble grows. When the buoyancy force overcomes the surface tension force the bubble is detached from the channel opening and starts to rise. This instant is detected by the linear CCD sensor. During the rise the bubble crosses the second branch of the laser beam, which detects its presence. At this point, it is possible to calculate the bubble rise velocity. It is then used to determine the instantaneous position of the bubble inside the chamber during its rise. When the bubble reaches the required distance from the wall, the actuator is initiated to provide the pressure pulse to explode the bubble.

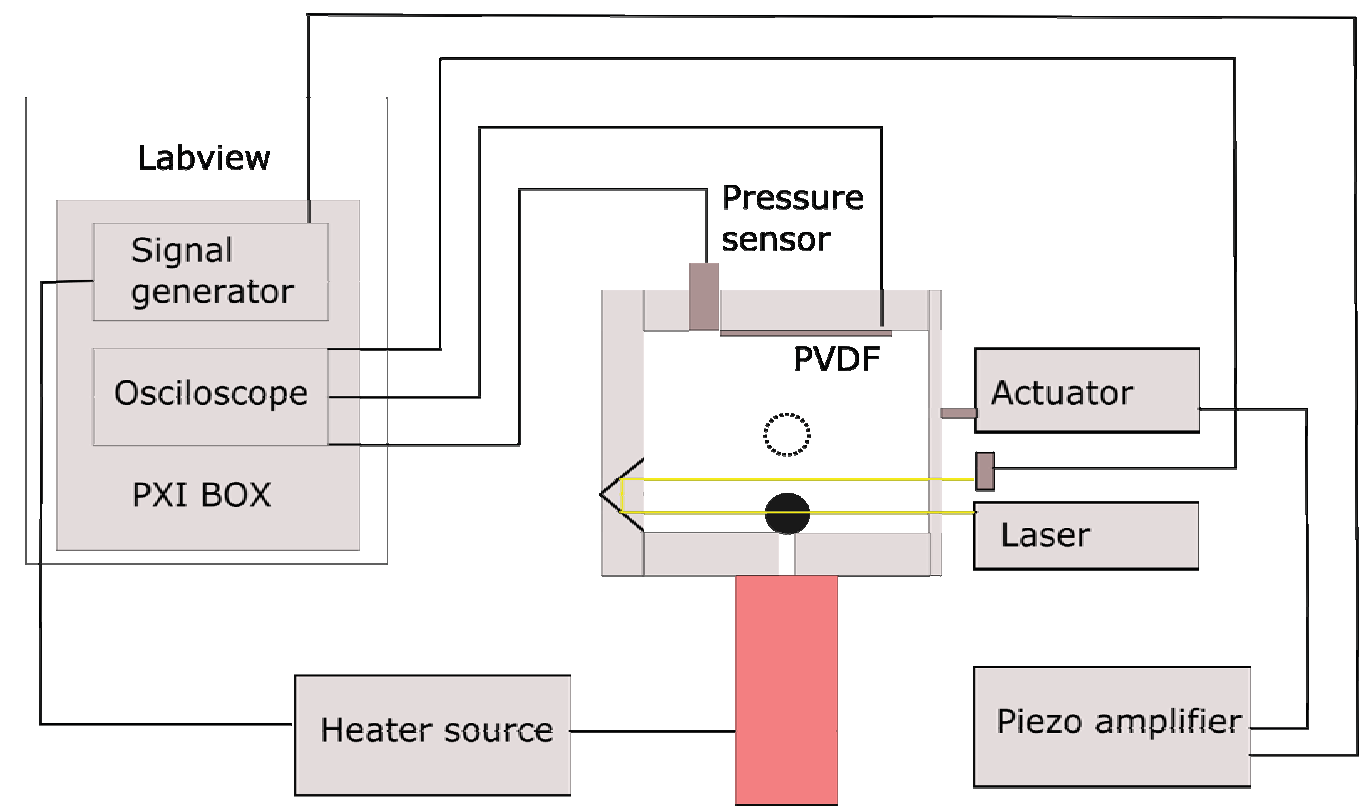

Figure 2. Measurement scheme 
Initially, the actuator assembly moves away from the chamber. During this cycle, the pressure inside the chamber is reduced to a required value controlled by the pressure sensor and monitored by the LabView software. During the second phase the actuator moves towards the chamber, increases the liquid pressure to initiate bubble implosion. Finally, the impact force of the bubble collapse is measured by the PVDF pressure film.

\section{Conclusion}

The experimental setup for the investigation of the cavitation bubble collapse has been designed. The setup allows to generate, excite and study bubbles collapsing near a solid wall in water. The system enables to measure the pressure impact exerted on the solid surface by the collapsing bubble. In the next step of the design, the system will be enhanced to control the air contamination of water, which could influence the measurement.

\section{ACKNOWLEDgemENT}

The authors wish to thank the Czech Science Foundation for providing financial support for grant no. 101/10/1428 and grant no. MSM 4674788501 of the Technical University of Liberec.

\section{References}

[1] Franc J. P., Michel, J. M.: Fundamentals of Cavitation, Kluwer Academic Publishers, 2004

[2] Vogel A., Lauterborn W., Timm R.: Optical and acoustic investigations of the dynamics of laser-produced cavitation bubbles near a solid boundary, J Fluid Mech 206, 1989, pp. 299-338

[3] Zima P., Sedlář M., Müller M.: Modeling collapse aggressiveness of cavitation bubbles in hydromachinery, Proc. of the 7th Intl. Symp. on Cavitation (CAV2009), Ann Arbor, Michigan, USA, 2009, paper \#131

[4] Wang Yi. C., and Chen Yu. W.: Application of piezoelectric PVDF film to the measurement of impulsive force generated by cavitation bubble collapse near a solid boundary, Exp. Thermal. Fluid Sci. 32, 2007, pp. 403-414 